Please quote as: Koene, P.; Felix, K.; Maximilian, K.; Marco, L. J. \& Helmut, K. (2012): Entwicklung und Evaluierung einer exemplarischen mobilen IKT-Anwendung zur Vermittlung von sozialen Internetdiensten. In: Technologiegestützte Dienstleistungsinnovation in der Gesundheitswirtschaft. Hrsg./Editors: Shire, K. A. \& Leimeister, J. M. Erscheinungsjahr/Year: 2012. Seiten/Pages: 137-157. 


\title{
Entwicklung und Evaluierung einer exemplarischen mobilen IKT-Anwendung zur Vermittlung von sozialen Internetdiensten
}

\author{
Philip Koene, Felix Köbler, Maximilian Könnings, Jan Marco Leimeister, \\ Helmut Kremar ${ }^{l}$
}

\section{Einleitung}

Soziale Netzgemeinschaften - engl. social networking sites (SNS) - wie Facebook, LinkedIn und Google, sowie die auf diesen bereitgestellten Internetdienste, erleben in den letzten Jahren einen enormen Anstieg in der Adoption und Nutzung. Die Motivationshintergründe, die zu einer Anmeldung, aktiven Teilnahme und Nutzung von bereitgestellten Internetdiensten führen, sind verschiedenartig (Bilandzic et al. 2009). Die primären Funktionalitäten dieser Plattformen sind jedoch das Verwalten von realweltlichen und virtuellen sozialen Verbindungen zu anderen Nutzern sowie die Bereitstellung von verschiedenen Funktionen zur Nutzung von Internetdiensten, bspw. das Einloggen an einem bestimmten Ort. Diese sozialen Verbindungen und durch spezifische Funktionen bereitgestellten Dienste, schaffen ein Unterstützungsnetzwerk und erzeugen unterschiedliche Arten von sozialem Kapital (Lampe et al. 2007; Leimeister et al. 2008).

Im Gegensatz zu früheren Generationen sozialer Netzgemeinschaften - sog. Virtuellen Communities - werden soziale Beziehungen in SNS typischerweise nicht virtuell und auf beiderseitigem Interesse initiiert (social browsing) (Lampe et al. 2006) und anschließend in realweltliche Beziehungen zwischen den Nutzern überführt (Parks/Floyd 1996; Parks/Roberts 1998; Cummings et al. 2002). Die aktive Teilnahme an SNS ist vielmehr durch eine Intensivierung und Verfestigung bestehender realweltlicher Beziehungen motiviert (social searching) (Lampe et al. 2006). Eine detaillierte Diskussion zu den Unterschieden zwischen den Nutzungsarten social searching und social browsing findet sich in Lampe et al. (2006). Obwohl etablierte SNS eine Vielzahl von Funktionalitäten zur Pflege und Stärkung sozialer Beziehungen unterstützen sowie eine Vielzahl an Internetdiensten bereitstellen, muss dennoch ein erheblicher Aufwand betrieben werden, um realweltliche Beziehungen auf SNS abzubilden oder Internetdienste abzurufen. So ergeben sich zahlreiche Nachteile für den Nutzer, bspw.

1 Die Autoren sind Mitarbeiter im BMBF-geförderten Forschungsprojekt Mobil 50+ (Förderkennzeichen: 01FC08046-8). 
eine signifikante zeitliche Diskrepanz zwischen der Knüpfung einer Bekanntschaft in einer realweltlichen Situation und deren virtueller Abbildung.

Dieser Medienbruch zwischen realweltlicher Situation und virtueller Abbildung, in Kombination mit mangelnder Benutzerfreundlichkeit und einer Nichtberücksichtigung der speziellen Bedürfnisse einer älteren Zielgruppe (Hawthorn 2003) haben auch dazu geführt, dass die Gruppe der Senioren die kleinste Nutzerschicht der SNS darstellt. Ein Mangel an sozialen Kontakten und an der Teilnahme an sozialen Aktivitäten erhöht für die ältere Generation das Risiko in die Pflegebedürftigkeit einzutreten (Stuck et al. 1999). SNS können einer älteren Bevölkerungsschicht Zugriff auf kulturelle und kommerzielle Angebote und Dienstleistungen, sowie neue Kommunikationsmöglichkeiten bieten und somit das Risiko der sozialen Isolierung vermindern (Craig 2004). Mobile Endgeräte ermöglichen dabei theoretisch einen zeit- und ortsunabhängigen Zugriff auf SNS (Leimeister et al. 2004).

Der NFriendConnector Prototyp versucht den Medienbruch zwischen realweltlicher Situation und virtueller Repräsentation zu verringern, und die realweltliche soziale Interaktion durch eine mobile, ubiquitäre Nutzerschnittstelle zu bereichern. Der Prototyp kann somit als exemplarisches Interaktionskonzept für andere Internetdienste dienen, die auch von einer älteren Generation leichter verstanden und genutzt werden können.

In diesem Beitrag wird der NFriendConnector als prototypische Anwendung anhand des "Uiqubitous Computing Application Development and Evaluation Process Model" (UCAN) (Resatsch 2010) entwickelt. Die Arbeit beschreibt die Reaktionen potentieller Nutzer gegenüber der prototypischen Anwendung und diskutiert Implikationen und Ergebnisse eines Laborexperiments.

\section{Methodik}

\subsection{Design Science}

Design Science kann als technologie-orientierter Forschungsansatz verstanden werden, der es versucht, Dinge zu erschaffen, welche menschlichen Zwecken dienen und diese anhand von Kriterien wie Wert und Nutzen zu beurteilen. Im Gegensatz zu traditionellen Naturwissenschaften zielt Design Science darauf ab, Lösungen und Möglichkeiten zu entwickeln, die der Erreichung von menschlichen Zielen dienen. Ergebnisse (sog. Artefakte) von Forschungsarbeiten, die nach dem Design Science Paradigma durchgeführt werden, sind entweder Konstrukte, Modelle, Methoden oder Instanziierungen (bspw. die prototypische Anwendung NFriendConnector).

Es gibt zwei grundlegende Aktivitäten, die jede Design Science Forschungsarbeit einschließen - die Erstellung und die Evaluierung eines Artefakts. Hevner 
(2007) lässt diese beiden Aktivitäten in einen iterativen „design cycle“ einflieBen (siehe Abbildung 1), der die Erzeugung und Evaluierung von Alternativen erlaubt, bis ein zufriedenstellendes Artefakt gestaltet worden ist. In der Regel wird ein Artefakt in Bezug auf Funktionalität, Vollständigkeit, Konsistenz, Fehlerfreiheit, Leistung, Ausfallsicherheit und Bedienbarkeit evaluiert (Peffers et al. 2006). Die Anforderungen für ein Artefakt sind innerhalb des Anwendungsumfeldes zu identifizieren, um so die Grundlage für die Erstellung und Evaluierung des Artefaktes bereitzustellen. Somit ist es notwendig ein ausreichendes Verständnis (,relevance cycle") der sozio-technischen und organisatorischen Umweltfaktoren zu entwickeln. Zusätzlich wird im Kontext der gestaltungsorientierten Forschung gefordert, die entwickelten Artefakte durch geeignete Evaluierungsmethoden (bspw. Fokusgruppen und Experimentes) zu erforschen (,rigor cycle") und diese Ergebnisse zu dokumentieren (Hevner 2007).

Die Entwicklung und Evaluierung des NFriendConnector erfolgte in drei Schritten, die sich an der gestaltungsorientierten Forschung ausrichtete: (1) Erarbeitung der Problemrelevanz; (2) Gestaltung des Artefakts mit Hilfe der Richtlinien zur Entwicklung von ubiquitären Anwendungen nach Resatsch (2010) und (3) quantitative Evaluierung des Artefakts nach der Expectation Confirmation Theory (ECT) (Oliver, 1980).

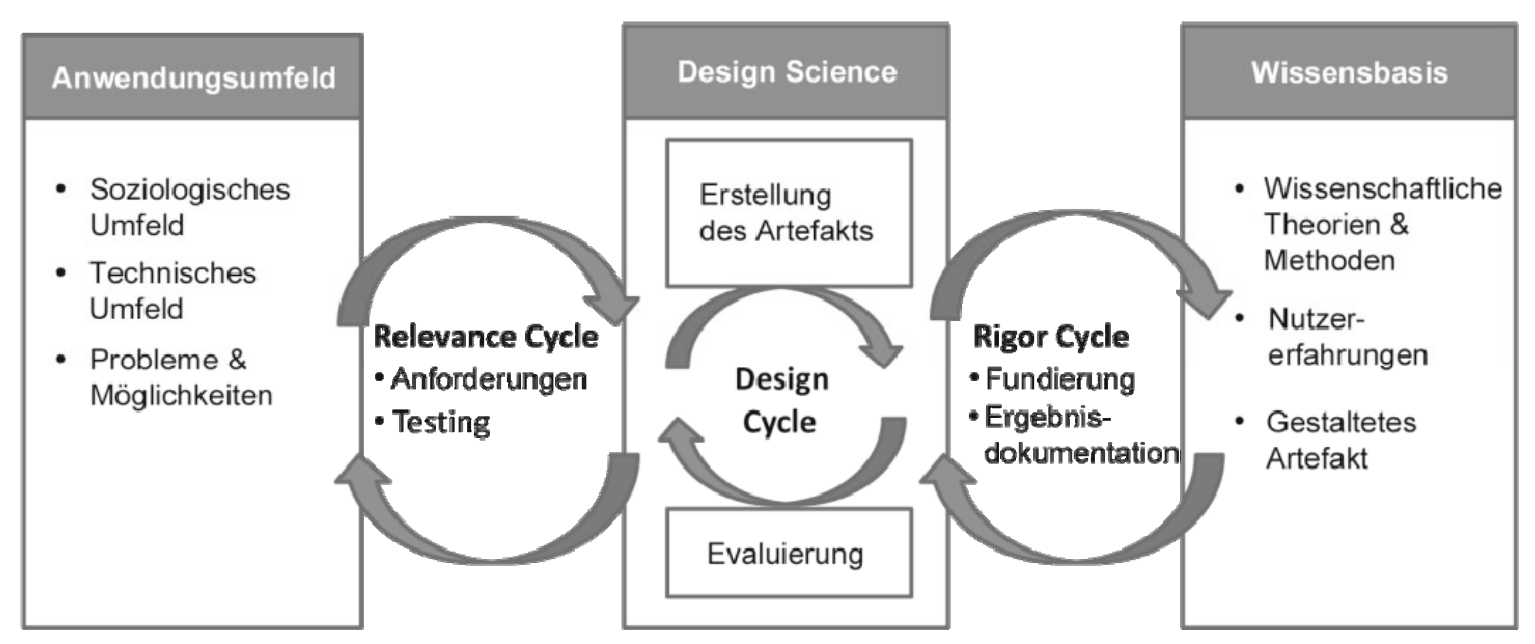

Abbildung 1 Gestaltungsorientierte Forschung nach dem Design Science Paradigma nach Hevner (Quelle: Eigene Darstellung in Anlehnung an (Hevner 2007))

\subsection{Ubiquitous Computing Application Development and Evaluation Process Model (UCAN)}

Die iterative Natur der Zyklen des Design Science Forschungsansatzes fordert ein iterativ-ausgerichtetes Entwicklungsmodell für mobile, ubiquitäre Anwendungen. Die Entwicklung des NFriendConnector Prototyps wurde daher anhand 
des "Ubiquitous Computing Application Development and Evaluation Process Model" (UCAN) (Resatsch 2010) umgesetzt. Abbildung 2 stellt die Phasen der verschiedenen Iterationsstufen der Entwicklung einer prototypischen mobilen, ubiquitären Anwendung auf Basis des UCAN Modells dar.

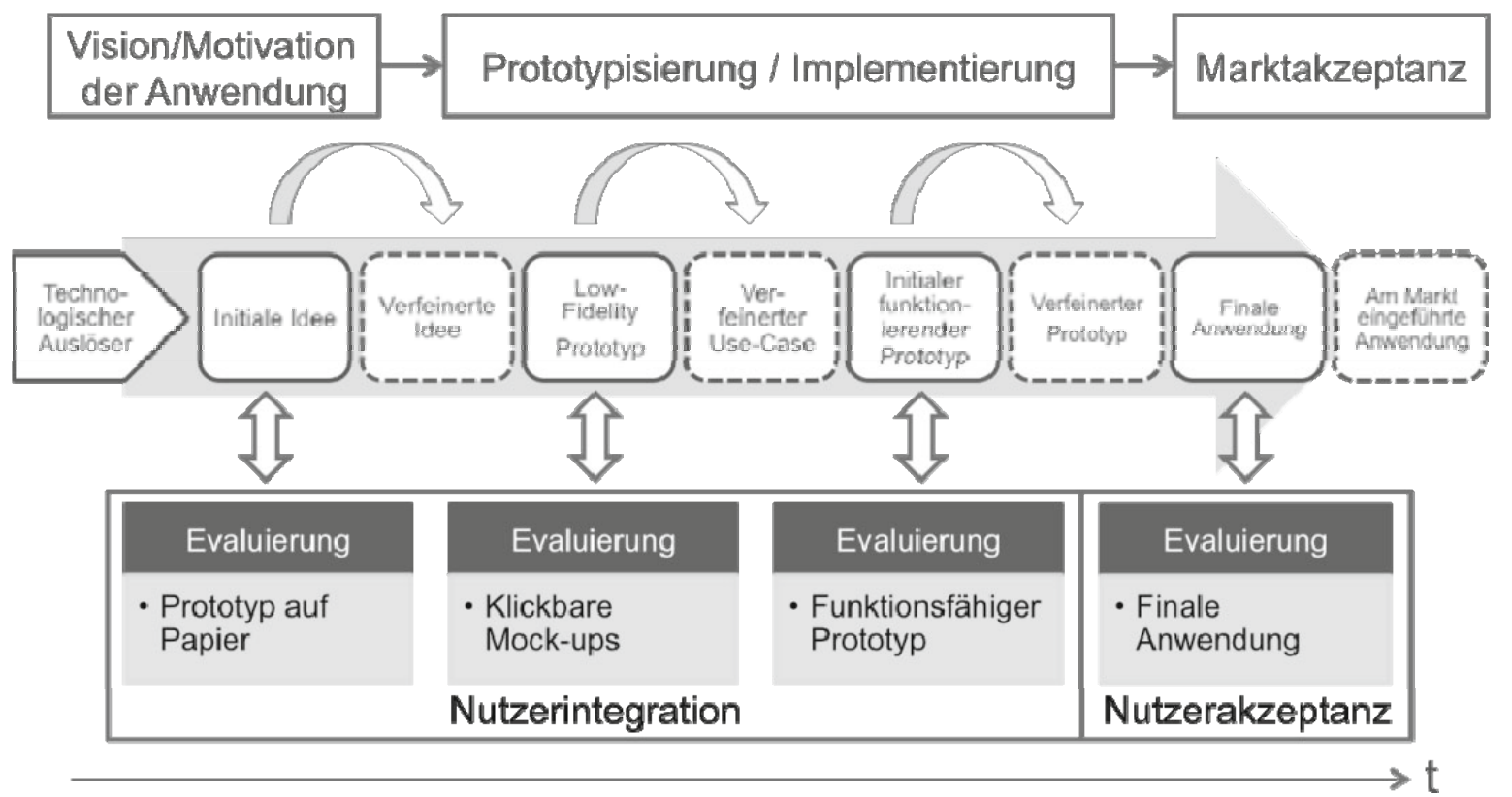

Abbildung 2 UCAN Vorgehensmodell (Quelle: Eigene Darstellung in Anlehnung an (Resatsch 2010))

Im Fall der Entwicklung des NFriendConnector ist der technologische Auslöser die Integration der Technologie Near Field Communication (NFC) in mobile Endgeräte und eine dadurch ermöglichte mobile, ubiquitäre Nutzung von SNS Funktionen und Internetdiensten, die in einer potentiellen Verbesserung der Interaktion und Nutzung dieser Dienste resultieren. Jeder Entwicklungsschritt wurde durch eine Evaluierung abgeschlossen. Initial wurden prototypisch Benutzeroberflächen auf Papier (paper-based mock-ups) erstellt und diese durch zwei Fokusgruppen $(\mathrm{N}=10)$ diskutiert und bewertet. Mit den hieraus gewonnen Erkenntnissen konnten die prototypischen Benutzeroberflächen zu interaktiven Prototypen (low-fidelity prototype) verfeinert werden. Die Evaluierungsergebnisse erlauben es im Anschluss daran, einen verfeinerten Anwendungsfall (refined use case) zu erstellen und diesen in einen voll funktionsfähigen Prototypen (high-fidelity prototype) zu überführen. Die in diesem Beitrag dargestellten Ergebnisse der Evaluierung fokussieren auf die Nutzerakzeptanz einer „finalen Anwendung“. 


\section{Verwandte Arbeiten}

Eine Vielzahl von Forschungsprojekten beschäftigt sich mit der prototypischen Entwicklung von mobilen, ubiquitären Anwendungen, die auf Funktionalitäten die durch SNS bereitgestellt werden, oder ähnlichen Internetdiensten zurückgreifen. Diese Anwendungen greifen meist auf Technologien, wie bspw. Bluetooth, Global Positioning System (GPS), Wireless Local Area Network (WLAN) zum Zwecke der Annäherungs- und Ortserkennung zurück und werden in der Literatur meist als social proximity Anwendungen (Eagle und Pentland 2005; Persson et al. 2005; Ziv und Mulloth 2006) bezeichnet. Im Gegensatz zu einigen Ausnahmen (Kostakos und O'Neill 2008; Pietiläinen et al. 2009) basieren die meisten Anwendungen auf kleinen, proprietären sozialen Netzwerkplattformen und blenden dabei die enorme Nutzerbasis sowie den Umfang an bereitgestellten Funktionen und Diensten der etablierten SNS aus. Aus der technologischen Umsetzung dieser Anwendungen ergeben sich für den Nutzer datenschutzrechtliche Nachteile und negative Auswirkungen auf die Privatsphäre (Eagle und Pentland 2005; Kostakos und O'Neill 2008).

NFriendConnector bedient sich im Gegensatz zu diesen nicht an Informationen zu Orten, Entfernung zu anderen Nutzern, oder vergleichbaren sensorischen Daten. Der Prototyp ermöglicht die nahtlose Integration der sozialen realweltlichen Interaktion in SNS durch die Benutzung eines Mobiltelefons mit ausgestatteter NFC-Schnittstelle. Dabei muss die Interaktion und der Austausch von Information zwischen zwei Nutzern von den Nutzern selbst willentlich initiiert werden, indem diese einen nahezu physischen Kontakt zwischen den mobilen Endgeräten herstellen. Folglich führt die Anwendung keinen Datenaustausch ohne die aktive Beteiligung der Nutzer aus und das Konzept dieser Anwendung steht exemplarisch für einen sichere Vermittlung und Imitierung von Internetdiensten.

\section{NFriendConnector Anwendung}

\subsection{Problemdefinition}

Zum besseren Verständnis eines möglichen Nutzungsszenarios und der Problemdefinition dient folgende exemplarische Beschreibung eines Anwendungsfalles:

Seit dem letzten Treffen zwischen Andrea und Michael ist einige Zeit vergangen, da Michael nach dem Eintreten in den Ruhestand in eine andere Stadt gezogen ist. Nach Jahren ohne persönlichen Kontakt treffen sich die beiden ehemaligen Arbeitskollegen auf einer Geburtstagsfeier eines gemeinsamen Freundes. An- 
drea und Michael verfügen über ein NFC-fähiges mobiles Endgerät mit einem mobilen Breitbandanschluss, der installierten NFriendConnector Anwendung und sind registrierte Nutzer der Facebook Netzwerkplattform. Die Anwendung ermöglicht den Austausch ihrer Profildaten über die NFC-Schnittstelle, indem die Mobiltelefone für eine kurz Zeit zusammen gehalten werden. Nach erfolgreicher Übermittlung können beide die Profildaten des jeweils anderen betrachten und mit ihren eigenen vergleichen. Auf dieser Grundlage entdecken beide, dass sie das gleiche Hobby teilen und passionierte Schachspieler sind. Die Anwendung ermöglicht es ihnen nun augenblicklich eine Facebook Freundschaftsbeziehung zu erstellen. Michael will seinen virtuellen Freundeskreis von diesem Wiedersehen mit Andrea unterrichten und generiert eine automatisierte Statusnachricht, die über das Treffen informiert und auf seiner Profilseite angezeigt wird.

Es kann viele Gründe für die Teilnahme und Nutzung einer SNS und der dort angebotenen Dienste geben. SNS ermöglichen es ihren Mitgliedern viele verschiedene menschliche Bedürfnisse zu adressieren, wie bspw. Kommunikation, Neugier und Selbstdarstellung. Dies geschieht durch die Erzeugung, den Austausch und die Kombination von Informationen (Bilandzic et al. 2009). Vorteile von der Teilnahme an SNS sind unter anderem der Zugang zu einer Vielzahl von Ressourcen, ein größeres soziales Kapital, sowie eine Zunahme an emotionaler, psychologischer und informationsbezogener Unterstützung (House 1981; Wellman et al. 2001; Lampe, Ellison und Steinfield 2007; Leimeister et al. 2008).

Während soziale Interaktionen im realen Leben spontan und natürlich geschehen, müssen Nutzer von SNS zurzeit noch enormen Aufwand betreiben (bspw. die Suche nach sozialen Kontakten und das Versenden von Freundschaftanfragen), um diese natürliche soziale Interaktion auf der SNS abzubilden. Dieser Aufwand tritt verstärkt zutage, wenn die Nutzer unerfahren im Umgang mit SNS sind, wie dies bspw. bei der älteren Generation gehäuft auftritt. Folglich existiert eine Lücke zwischen der realweltlichen Interaktion und Kontaktaufnahme und der virtuellen Abbildung dieser auf einer SNS. Diese Lücke kann auch auf die Initiierung und Vermittlung von Internetdiensten übertragen werden.

NFriendConnector schließt diese Lücke exemplarisch, indem die prototypische Anwendung am Beispiel von SNS, ein adäquates Nutzungskonzept präsentiert, welches die realweltliche und virtuelle soziale Interaktion verbindet.

\subsection{Prototypendesign}

Die Entwicklung richtet sich zum einen an dem bereits beschrieben UCAN Modell und zum anderen an den NFC-Design Richtlinien von Resatsch (2010) 
aus. NFC ist eine hochfrequente drahtlose Kommunikationstechnologie mit sehr geringer Reichweite, die unter Anderem einen Datenaustausch zwischen Endgeräten bis zu einer Entfernung von ca. $10 \mathrm{~cm}$ ermöglicht, und kompatibel zu Radio Frequency Identification (RFID) ist. NFC ist eine vergleichsweise neue drahtlose Kommunikationstechnik, die vor allem für mobile Anwendungen und Initiierung von Dienstleistungen (wie z. B., Bezahl- und Ticket-Anwendungen (Ondrus und Pigneur 2007; Mulliner 2009)) verwendet wird und dabei primär in mobilen Endgeräten zum Einsatz kommt.

Die Technologie ermöglicht somit eine Modalität der Interaktion, die einer Berührungsmetapher gleich kommt und so ein natürliches Konzept der Initiierung von Funktionen oder Internetdiensten darstellt. Die Nutzungsszenarien der prototypischen Anwendung basieren auf zwei NFC-fähigen Endgeräten, die mit einer mobilen Breitbandanbindung sowie einer ausreichenden Displayauflösung und -größe ausgestattet sind.

Die mobile Anwendung integriert eine Reihe von Funktionen der sozialen Netzwerkplattform Facebook in das mobile Endgerät durch die Benutzung der Facebook Application Programming Interface (API) und Nutzerprofildaten. Die Anwendung erlaubt Nutzern somit eine realweltliche soziale Interaktion zum Zeitpunkt des Geschehens, durch das Zusammenführen oder durch Berührung der mobilen Endgeräte, in eine virtuelle Interaktion zu übertragen.

Um einen Austausch von Profildaten zu initiieren stellen zwei Nutzer einen physischen Kontakt ihrer Mobiltelefone her, auf denen NFriendConnector läuft. Die Facebook Profildaten werden übertragen und der Nutzer kann über das Initiale Menü (siehe Abbildung 3) auf die im Folgenden beschriebenen Funktionen zugreifen.

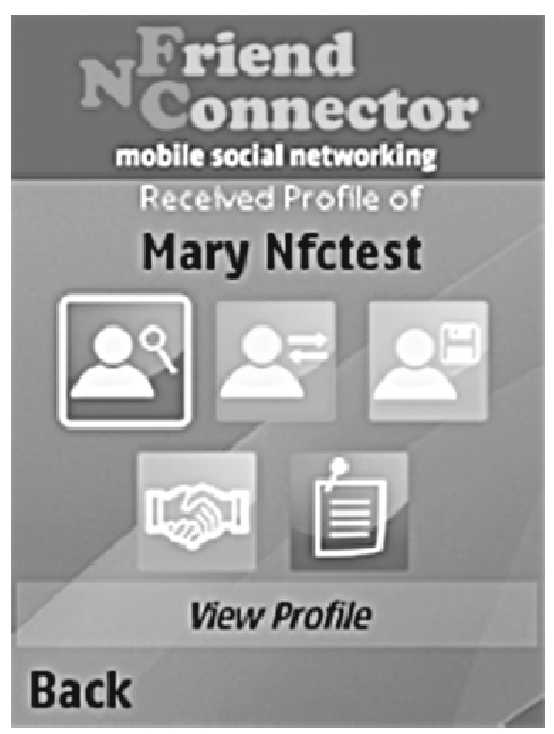

Abbildung 3 „Profil Optionen“ Menü 
Der Nutzer kann nun die Profildaten der anderen Person auf seinem Mobiltelefon ansehen und lokal speichern. Im NFriendConnector Prototyp werden nur das Profilbild, der Name und die Felder Heimatstadt, Interessen, Filme und Musik angezeigt (siehe Abbildung 4). Es ist jedoch technisch möglich, alle vorhandenen Profildaten anzuzeigen. Das lokale Speichern der Profildaten erlaubt es dem Anwender eine individuelle Kontaktliste seiner virtuellen Beziehungen auf seinem Mobiltelefon zu erstellen, die später auch ohne eine aktive mobile Internetverbindung zur Verfügung steht.
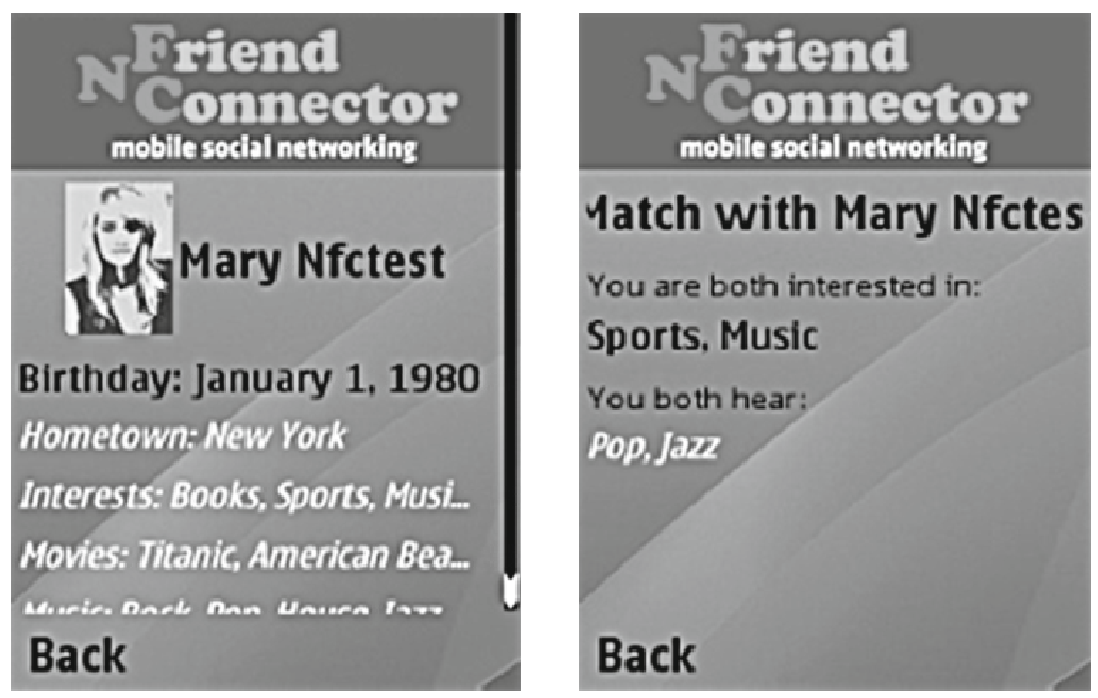

Abbildung $4 \quad$ "Profil betrachten" und "Profil vergleichen" Funktionen

Die "Profil vergleichen" Funktion ermöglicht es dem Nutzer, sein eigenes Facebook-Profil mit dem Facebook-Profil einer anderen Person zu vergleichen, um gemeinsame Interessen, Vorlieben und Abneigungen, Hobbies, etc. zu identifizieren (siehe Abbildung 4). Diese kann bei der Partnersuche, oder bspw. bei der Suche nach Teilnehmern für gemeinschaftliche Veranstaltungen (z.B. Sport) hilfreich sein. Der NFriendConnector Prototyp vergleicht alle Profildaten.

NFriendConnector ermöglicht den Nutzern auch den Zugriff auf die Facebook Funktionen ,als Freund hinzufügen" und " Statusmeldung erstellen" (siehe Abbildung 5). Ein einfaches Beispiel für eine solche, automatisierte Statusmeldung ist " $<$ Person $1>$ ist an $<$ Ort $>$ mit $<$ Person2 $>$ " (siehe Abbildung 5). Der Ort (in diesem Fall "Monaco, Frankreich") kann durch den GPS-Empfänger des Mobiltelefons abgefragt werden. 


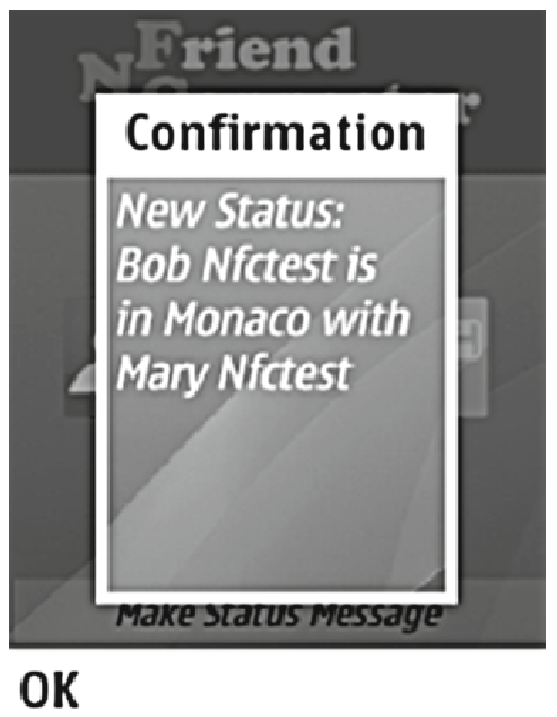

Abbildung 5 „Statusmeldung erstellen“ Funktion

\subsection{Implementierung der NFriendConnector Anwendung}

Der NFriendConnector Prototyp wurde für das NFC-fähige Mobiltelefon Nokia 6212 classic entwickelt. Anwendungen, die auf NFC Technologie zurückgreifen, benutzen eine von drei unterschiedlichen Betriebsarten, die wie folgt, definiert sind (Forum 2010):

- Der Peer-to-Peer Modus wird für eine bidirektionale Kommunikation zwischen zwei NFC-fähigen Endgeräten verwendet. Dieser Modus ermöglicht die Übertragung von kleinen Datenmengen zwischen mobilen Endgeräten, z.B. Kontakt- und Kundendaten (Köbler et al. 2010) oder Daten, die für die Initiierung sowie der sicheren Abrechnung von (personenbezogenen) Dienstleistungen verwendet werden können.

- In dem Emulationsmodus agiert ein NFC-fähiges Endgerät wie eine Smartcard, die dann von einem externen NFC-Lesegerät gelesen werden kann. Dieser Modus wird primär für die Bereitstellung von Bezahldiensten verwendet (Ondrus und Pigneur 2007).

- Der Lese/Schreib-Modus erlaubt einem NFC-fähigen (mobilen) Endgerät Daten von einem NFC-konformen, passiven (ohne Batterie) Transponder auszulesen (Madlmayr et al. 2008). Diese Transponder oder Tags können Informationen, bspw. Standortdaten beinhalten (Köbler, et al. 2010; Koene et al. 2010) und bieten somit enormes Potential zur Initiierung von Dienstleistungen oder den Zugriff auf Internetdienste. 
Die NFriendConnector Anwendung ist als Java J2ME Midlet implementiert, welches einerseits die NFC-Schnittstelle des mobilen Endgeräts ansprechen und andererseits auf die Funktionen der SNS Facebook zugreifen kann. Die Kommunikation mit der Facebook Netzwerkplattform wird über REST HTTP Anfragen an den Facebook API REST Server über die mobile Internetanbindung realisiert. Die NFC-Lese-/Sendeeinheit des Nokia 6212 wird dabei von dem Java J2ME Midlet über eine API gesteuert, die von Nokia zur Verfügung gestellt wird. Der allgemeine Kommunikationsablauf ist exemplarisch in Abbildung 6 dargestellt.

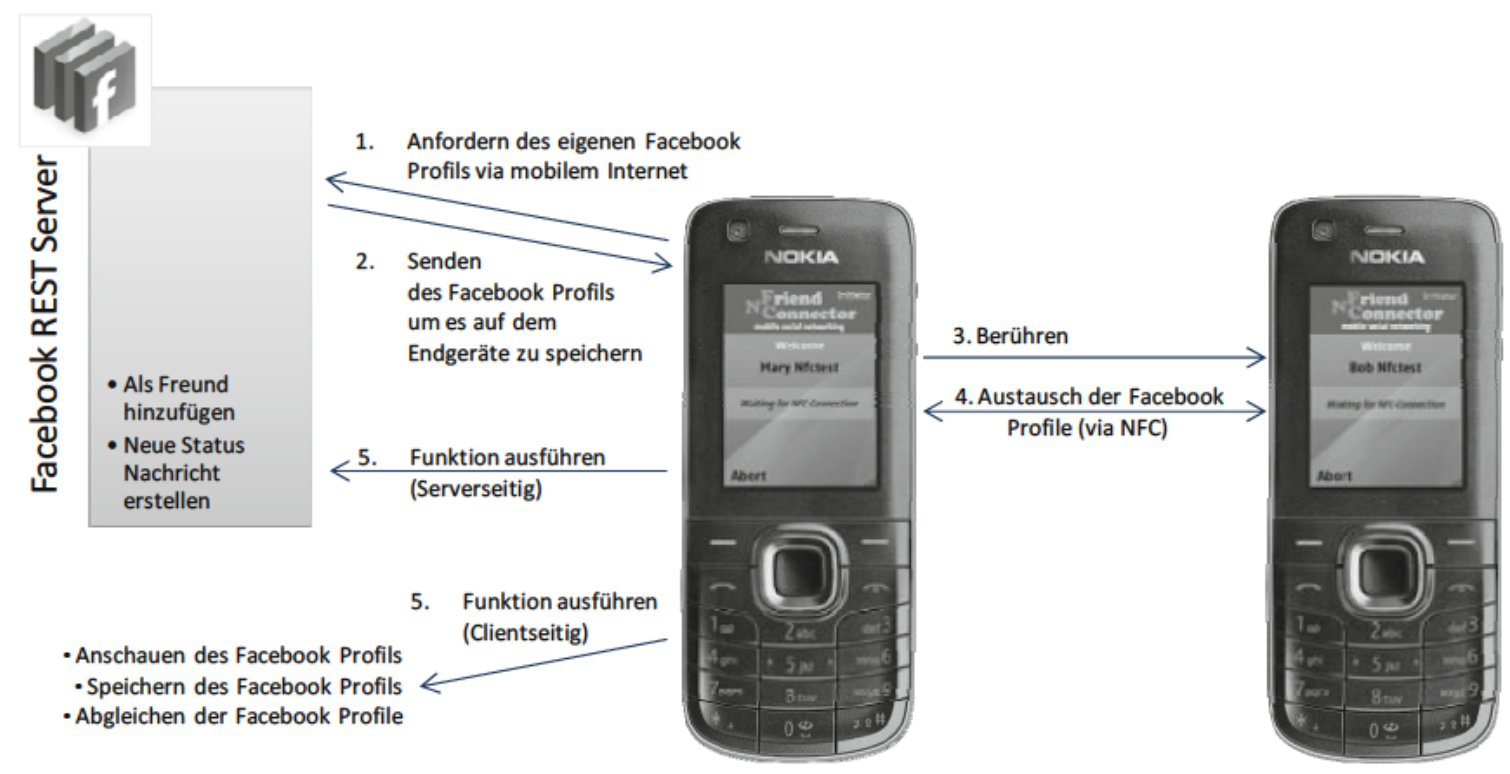

Abbildung 6 Der NFriendConnector Kommunikationsablauf (eigene Darstellung)

Für die prototypische Implementierung wurde eine Mischform der beiden vorgestellten Kommunikationsabläufe gewählt (Abbildung 6). Bei der gewählten Umsetzung kann der Nutzer lokal gespeicherte Profildaten nach jedem Start der Anwendung aktualisieren. Dabei werden nur Profildaten und keine Multimediadaten (wie bspw. Profilbilder) gespeichert. Um eine hohe Datenlast über die NFC-Schnittstelle zu vermeiden, werden die Profildaten als XML-Datei über die NFC-Schnittstelle ausgetauscht und Multimediadaten direkt über die mobile Internetanbindung heruntergeladen. Der direkte Zugriff auf Profildaten wird dabei durch den Facebook REST API Server ermöglicht, über welchen zudem Facebook Funktionen, durch HTTP GET/POST Nachrichten an den REST Server, benutzt werden können, ohne dabei einen mobilen Internetbrowser zu verwenden. Der Großteil der NFriendConnector Funktionen wurde technisch auf 
diese Weise realisiert, jedoch können einige Facebook Funktionen, wie bspw. der Login-Prozess, noch nicht über den Facebook REST API Server angesprochen werden. Zur Realisierung dieser Funktionen öffnet die Anwendung automatisch den mobilen Internetbrowser, der die entsprechende mobile Internetseite anzeigt, bspw. Facebook Login. Der Nutzer kann sich über diese an der Plattform anmelden und gelangt durch das Schließen des mobilen Internetbrowsers zur NFriendConnector Anwendung zurück. Die gewählte Realisierung ist aufgrund der momentan mangelhaften Kompatibilität der Facebook REST API mit mobilen Endgeräten notwendig, diese soll jedoch in naher Zukunft durch Initiativen, wie bspw. Facebook Platform for Mobile verbessert werden.

\section{Evaluierung}

NFriendConnector wurde durch ein Laborexperiment und einer anschließenden Befragung der Probanden evaluiert. Durch das Laborexperiment sollte einerseits abgeprüft werden, welche Intentionen hinter der Nutzung von SNS stehen und andererseits inwieweit die Probanden die Anwendung in der Verwendung nützlich finden, und die Anforderungen zur Gestaltung von mobilen, ubiquitären Anwendungen erfüllt wurden, die auch auf zukünftige Anwendungen zur Initiierung und Abrechnung von Internetdiensten sowie (personenbezogener) Dienstleistungen, angewandt werden können. Die Zufriedenheit mit der Nutzung einer IKT-Anwendung (Informations- und Kommunikationstechnologie) ist ein anerkannter Indikator für die Absicht diese auch (zukünftig) zu nutzen. Im den folgenden Kapitel werden das verwendete theoretische Rahmenwerk zur Evaluierung und das Experimentdesign beschrieben sowie die Ergebnisse diskutiert.

\subsection{Expectation Confirmation Theorie (ECT)}

Die Auswahl von geeigneten Theorien und Methoden zur Evaluierung eines entworfenen Artefaktes ist ein wesentlicher Bestandteil des Design Science Forschungsansatzes (Hevner 2007). Ein zentraler Gradmesser für Akzeptanz einer Anwendung ist die Nutzerzufriedenheit (Vasalou et al. 2010). Dementsprechend haben wir uns für die Evaluierung der NFriendConnector Anwendung, die beispielhaft für eine ubiquitäre Anwendung zur Nutzung von Internetdiensten steht, für die Expectation Confirmation Theorie (ECT) (1993) entschieden. Die ECT kann, die neben etablierten Theorien, wie bspw. das Technology Acceptance Model (TAM) (Davis et al. 1989; Venkatesh und Davis 2000; Venkatesh et al. 2003), und in verschiedenen Varianten (1993; 1999; 2002; 2010; 2011), zur Evaluierung von IKT-Anwendungen, eingesetzt werden. Die Theorie entstammt der Konsum- und Marketingforschung, um Verbraucherzufriedenheit und Kaufentscheidungen vorauszusagen. Prinzipiell basiert die ECT auf der Differenz zwischen den vorherigen Erwartungen der Benutzer an ein jeweiliges Produkt, IKT-Anwendung oder Dienstleistung und der Erwartungsbe- 
stätigung bzw. -widerlegung (Oliver 1980; Swan und Trawick 1981; Tse und Wilton 1988; Anderson und Sullivan 1993; Pizam und Milman 1993; Spreng et al. 1996; Patterson et al. 1997; Dabholkar et al. 2000). Oliver (1980) liefert eine ausführliche theoretische Abhandlung, Beschreibung und Diskussion zu Vorund Nachteilen der ECT. Das von uns als Forschungsmodell verwendete Expectation Confirmation Model (ECM) (Bhattacherjee 2001) (siehe Abbildung 7) misst die zuvor bestehende Akzeptanz in den Konstrukten Bestätigung (Confirmation) und Zufriedenheit (Satisfaction).

ECM verwendet darüber hinaus den kognitiven Faktor der wahrgenommenen Nützlichkeit (Perceived Usefulness) zur Vorhersage von Nutzungsabsichten (Intention to Use) (Bhattacherjee 2001). Das ECM sagt die Nutzungsabsicht für eine Anwendung, basierend auf deren Zufriedenstellung nach der erstmaligen Nutzung, vorher. Dabei wurden fünf Hypothesen aufgestellt, die die theoretischen kausalen Zusammenhänge zwischen den Konstrukten vorhersagen (siehe Abbildung 7, H1-H5) (Bhattacherjee 2001). Erwartungen können durch die Nutzung des IKT-Artefakts beeinflusst werden und sich verändern, bzw. verstärken sich im Regelfalle durch die Benutzung eines IKT-Artefakts (Bhattacherjee 2001).

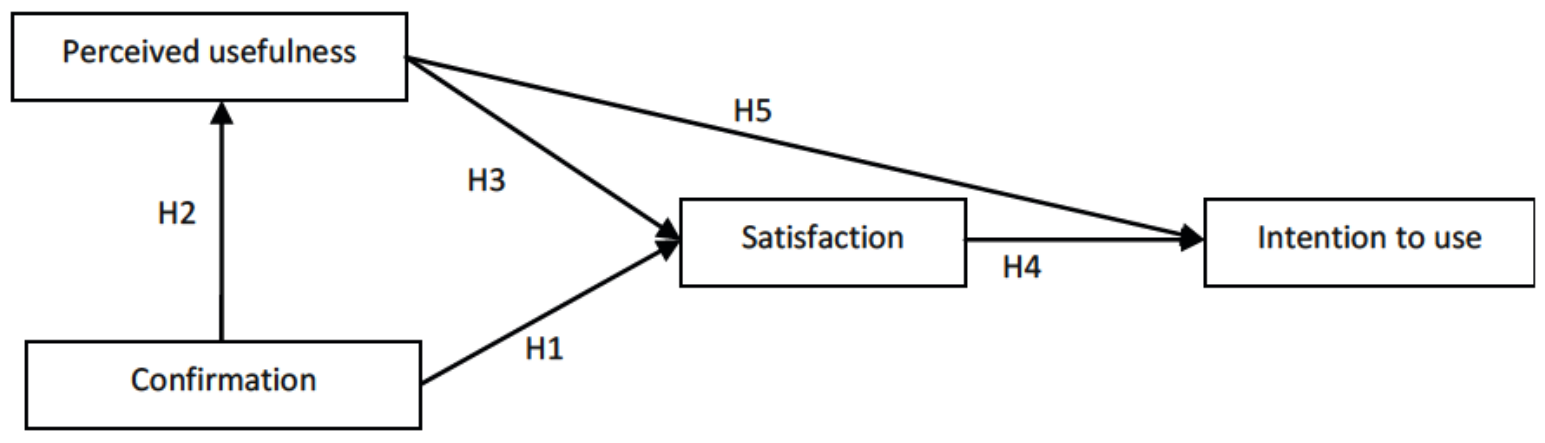

Abbildung $7 \quad$ Forschungsmodell (in Anlehnung an (Bhattacherjee 2001))

\subsection{Experimentdesign}

Eine experimentelle Methode wurde gewählt, um den NFriendConnector zu evaluieren. Für dieses Forschungsdesign ist ein Laborexperiment eine geeignete Methode, da es sie uns erlaubt, externe Faktoren zu kontrollieren. Um die externen Einflüsse des Experimentes so klein wie möglich zu halten, wurde das Experiment in einem einfachen Raum und mit denselben NFC-fähigen mobilen Endgeräten der gleichen Marke sowie Modell durchgeführt.

Das Laborexperiment wurde anhand eines festgelegten Protokolls durchgeführt nach dem die Probanden zunächst über die NFriendConnector Anwendung, die realisierten Funktionen und ein fiktives Nutzungsszenario informiert wurden. 
Anschließend wurden die Teilnehmer in Gruppen je zwei Personen aufgeteilt und gebeten die unterschiedlichen Funktionen der Anwendung, auf zwei NFCund internetfähigen Mobiltelefonen, zu testen. Dabei wurden Profildaten generischer Nutzer verwendet, die vor dem Laborexperiment in der SNS Facebook hinterlegt wurden. Insgesamt nahmen 62 Probanden an unserem Experiment teil, die während des Experimentes aufgefordert wurden zwei Fragebögen (ex ante / ex post der Nutzung) auszufüllen. Der erste Fragenbogen, welcher vor der Einführung des Prototyps auszufüllen war, nahm den demographischen Hintergrund der Probanden auf (bspw. Alter und Bildungsabschluss). Der zweite Fragenbogen, der nach der Nutzung der Anwendung aufzufüllen war, setzte sich aus den validierten Frageitems der wichtigsten ECT Variablen zusammen. (Davis, Bagozzi und Warshaw 1989; Bhattacherjee 2001). Die Erwartungen der Probanden gegenüber der Anwendung wurden dabei, wie bei der Verwendung von ECM üblich, ex post im zweiten Fragebogen abgeprüft.

\subsection{Ergebnisse und Diskussion}

Durch die Auswertung des Experiments zeigte sich, dass die Probanden SNS benutzen, um realweltliche und existierende Beziehungen zu anderen Personen zu verwalten, und nicht um reine und primär virtuelle Beziehungen zu initiieren und zu unterhalten.

Tabelle 1 zeigt die demographische Zusammensetzung des durchgeführten Experimentes. Insgesamt nahmen 62 Probanden an dem Experiment teil $(\mathrm{N}=62)$. Das Experiment wurde mit Nutzern einer jüngeren Zielgruppe durchgeführt, da ein Vorwissen im Umgang mit mobilen Endgeräten und sozialen Netzwerken hilfreich war. Da es sich jedoch bei der Interaktion mit NFriendConnector um eine intuitiv zu verstehenden Berührungsmetapher handelt, gehen wir davon aus, dass sich die Ergebnisse des Experiments im Sinne eines „Universal Design"- Ansatzes auch auf eine ältere Zielgruppe übertragen lassen (Plos und Buisine 2006). Ein angemeldeter Nutzer auf Facebook zu sein war jedoch keine Vorbedingung zur Teilnahme und die Daten zeigen, dass das Sample aus etwa zwei Dritteln Facebook Nutzern bestand.

Tabelle 1 Demographische Zusammensetzung des Experimentsamples

\begin{tabular}{|l|l|l|}
\hline $\begin{array}{l}\text { Demographische } \\
\text { Variable }\end{array}$ & Kategorien & Häufigkeit \\
\hline Alter & Jünger als 25 & $26(41,9 \%)$ \\
& $25-34$ & $35(56,5 \%)$ \\
& Älter als 35 & $1(1,6 \%)$ \\
\hline Geschlecht & Weiblich & $8(12,9 \%)$ \\
\hline
\end{tabular}




\begin{tabular}{|l|l|l|}
\hline & Männlich & $54(87,1 \%)$ \\
\hline Bildungsstand & Bachelor & $24(38,7 \%)$ \\
& Master & $26(41,9 \%)$ \\
& Promotion & $6(9,7 \%)$ \\
& Keine Angabe & $6(9,7 \%)$ \\
\hline Facebook Mitglied & Ja & $39(62,9 \%)$ \\
& Nein & $23(37,1 \%)$ \\
\hline $\begin{array}{l}\text { Mitglied einer } \\
\text { sozialen Netz- } \\
\text { werkplattform }\end{array}$ & Ja & $59(95,2 \%)$ \\
\hline
\end{tabular}

Da ein Drittel der Probanden Mitglieder bei anderen SNS waren, legen die Ergebnisse nahe, dass nicht nur Facebook, sondern SNS Nutzer generell zu dem beschriebenen Nutzungsverhalten tendieren. Dementsprechend ist anzunehmen, dass eine Anwendung, die es den Nutzern erlaubt, Daten in Echtzeit in einer realweltlichen sozialen Interaktion, mit einer Internetplattform auszutauschen, als sinnvoll und nützlich erachtet wird.

Unser Forschungsmodell wurde mittels einer Partial Least Squares (PLS) Pfadanalyse getestet, was eine gleichzeitige Beurteilung des Mess- und Strukturmodells ermöglicht. Das Messmodell wurde bezüglich Reliabilität, konvergenter Validität und diskriminanter Validität getestet. Abbildung 8 zeigt das Resultat der Pfadanalyse. Alle als Hypothesen aufgestellten Beziehungen waren statistisch signifikant.

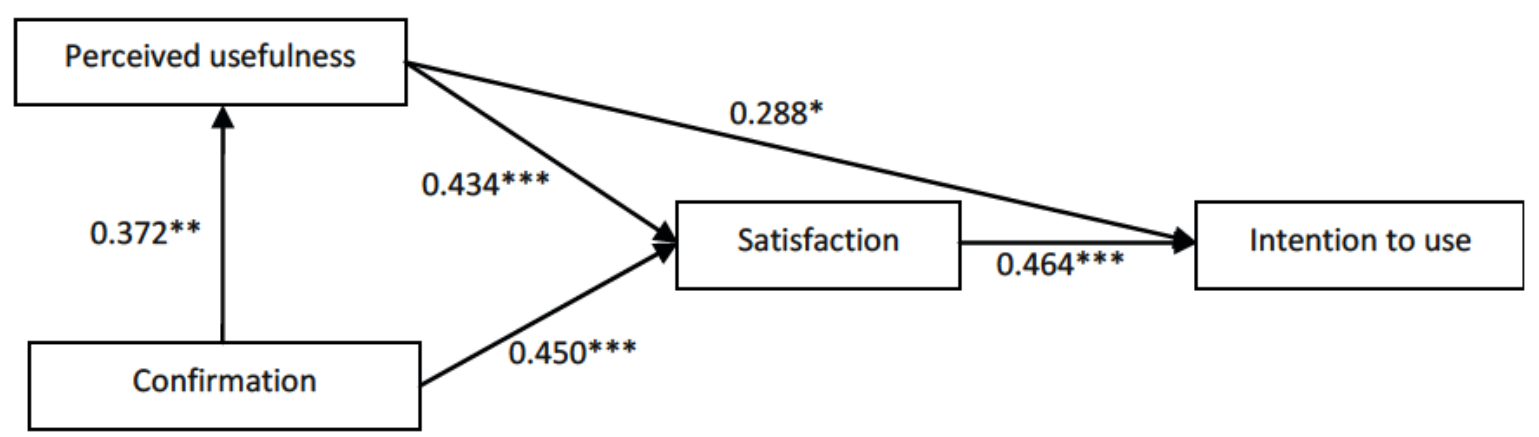

Abbildung 8 Ergebnisse der PLS Pfadanalyse auf den Signifikanz-Niveaus; ${ }^{*} \mathrm{p}<0.05 ; * * \mathrm{p}<0.01 ; * * * \mathrm{p}<0.001$ 
Es konnte zudem gezeigt werden, dass demographische Variablen, wie bspw. das Geschlecht und Alter keinen Einfluss auf die Antworten der Probanden hatten.

Eine gestaltungsorientierte Forschung, die als Beitrag ein IKT-Artefakt erzeugt, kann als erfolgreich angesehen werden, wenn die Zielgruppe der Benutzer die Anwendung als nützlich empfindet und bereit ist, diese auch zukünftig zu benutzen. Unsere Ergebnisse legen nahe, dass die Probanden NFriendConnector als nützliche Anwendung wahrgenommen haben. Es konnte zudem gezeigt werden, dass eine Gruppenzugehörigkeit (Facebook-Nutzer und nicht Facebook-Nutzer) keinen signifikanten Einfluss auf die Einstellung der Nutzer gegenüber der Anwendung hat.

Aktuelle Forschungsergebnisse und praktischen Entwicklungen im Bereich der SNS zeigen, dass es eine Verschiebung in den Verhaltensmustern der Nutzer von SNS gibt, hin zu einer engeren Verbindung zwischen sozialer Interaktion im offline Leben und sozialer Interaktion in SNS (Boyd 2004; Joinson 2008).

Technologische Entwicklungen wie Smartphones, Kommunikationstechnologien wie bspw. Bluetooth und NFC, sowie zunehmende Bandbreiten der mobilen Internetverbindungen ermöglichen es durch spezielle Anwendungen, Nutzer in realweltlichen Situationen zu unterstützen und ihnen bspw. das Initiieren von personenbezogenen Dienstleistungen über das Internet zu erleichtern.

\section{Limitierungen}

Die Funktionen der prototypischen Anwendung NFriendConnector sind stark auf die durch die SNS Facebook bereitgestellten Funktionen beschränkt. Dieser Sachverhalt könnte sich limitierend auf die Verallgemeinbarkeit der Ergebnisse des Experiments und der Reaktionen der Probanden auswirken. In nächsten Schritten können Experimente in realen sozialen Kontexten durchgeführt werden, eine Nutzerstudie über einen längeren Zeitraum durchgeführt werden bzw. das Konzept auf die Initiierung und Vermittlung von weiteren Internetdiensten und personenbezogener Dienstleistungen erweitert werden.

Die NFriendConnector Anwendung, sowie die durchgeführte Studie sollte im Rahmen ihrer Limitierungen interpretiert werden. NFriendConnector zeigt wie man einen Zugriff auf SNS Funktionen und Dienste in realen sozialen Interaktionen in Echtzeit auf einem NFC-fähigen mobilen Endgerät ermöglicht. Daher ist die derzeitige Umsetzung von NFriendConnector eine technische Machbarkeitsstudie, die sich spezifisch auf die SNS Facebook bezieht und NFC-fähigen Mobiltelefon benötigt. Darüber hinaus unterstützt NFriendConnector nur eine begrenzte Anzahl von Funktionalitäten, die über die SNS Facebook abgerufen werden können. Die Existenz und Popularität von kommerziellen Anwendungen 
(bspw. Bump, Google Latitude), die ähnliche Funktionen, wie die in diesem Beitrag vorgestellte prototypische Anwendung NFriendConnector, bieten, werden zunehmend zur Initiierung und Vermittlung von (personenbezogenen) Dienstleistungen oder zur Bezahlung von Produkten oder Dienstleistungen, eingesetzt.

\section{Zusammenfassung und Ausblick}

Die Umsetzung des gewählten Anwendungsszenarios für den NFriendConnector war primär durch die Erforschung der Nutzerakzeptanz und technische Machbarkeit einer auf NFC-basierenden prototypischen Anwendung motiviert, und sollte weniger potentielle kommerzielle Geschäftsfelder aufzeigen. Es sind jedoch verschiedene kommerzielle Anwendungsszenarien denkbar, die auf der bidirektionalen Kommunikation einer NFC-fähigen mobilen Anwendung, bspw. der Initiierung und Vermittlung von (personenbezogenen) Dienstleistungen, zurückgreifen.

Mobile Anwendungen, die durch ubiquitäre Technologien stärker in realweltliche Prozesse integriert werden können, resultieren in einer zunehmenden benutzerfreundlicheren Verwendung und Nutzung der auf Plattformen integrierten Funktionalitäten, bereitgestellten Diensten, sowie Dienstleistungen. Diese benutzerfreundlichere Verwendung, sowie die Reduzierung von Medienbrüchen kann die Technologieakzeptanz für SNS und Internetdiensten gerade für die ältere Generation erhöhen. Für Betreiber dieser Plattformen kann aus diesem generierten Mehrwert ein wirtschaftliches Potential abgeleitet werden. Ein mögliches Geschäftsmodell könnte bspw. auf einer monatlichen Grundgebühr, oder, im Sinne eines hybriden Produkts (Leimeister/Glauner 2008), auf der Initiierung und Vermittlung von (personenbezogenen) Dienstleistungen über eine Internetplattform beruhen.

Des Weiteren zeigt sich, dass es gerade in der ubiquitären und mobilen ITUnterstützung von Dienstleistungen an Modellierungsansätzen und Werkzeugunterstützung für Entwurf und Konstruktion von Dienstleistungssystemen mangelt. Dies gilt insbesondere für die Teilautomatisierung von Dienstleistungen, ermöglicht durch Sensordaten wie bspw. Ortsinformation durch GPSEmpfänger. Außerdem fehlen Service Prototyping Lösungen, mit denen ubiquitäre IT-Unterstützung von Dienstleistungen während der Entwicklung leichtgewichtig und schnell für Endanwender „erlebbar“ gemacht werden können.

Die Zielsetzung der vorgestellten prototypischen Implementierung war es folglich (1) ein neues, innovatives technisches Konzept zu beschreiben, welches die mobile und ubiquitäre Nutzung von Internetdiensten ermöglicht, durch (2) einen systematischen Entwicklungsprozess gestaltet und (3) durch wissenschaftliche Methoden evaluiert wurde. NFriendConnector und der systematische Entwick- 
lungsprozess kann dabei auch als neue, innovative Form der IT-gestützten Dienstleistung gesehen werden, bzw. als ein methodischer Vorschlag zum Service Engineering. Der Prototyp wurde als eine proof-of-concept Anwendung implementiert und basiert auf der SNS Facebook und NFC-fähigen mobilen Endgeräte und kann als Ausgangspunkt für vergleichbare Konzepte dienen, die die Initiierung und Vermittlung von personenbezogenen Dienstleistungen, unterstützen. 


\section{Literaturverzeichnis}

Anderson, E. W. / Sullivan, M. W. 1993: The Antecedents and Consequences of Customer Satisfaction for Firms. Marketing Science 12(2): 125-143.

Bhattacherjee, A. 2001: Understanding Information Systems Continuance: An Expectation-Confirmation Model. MIS Quarterly 25(3): 351-370.

Bilandzic, M. / Filonik, D. / Gross, M. / Hackel, A. / Mangesius, H. / Krcmar, H. 2009: Mobile Application to Support Phatic Communication in the Hybrid Space. 6th International Conference on Information Technology: New Generations, Las Vegas, USA: IEEE Computer Society.

Boyd, D. M. 2004: Friendster and publicly articulated social networking. CHI '04 Human factors in computing systems, Vienna, Austria: ACM.

Brown, S. A. / Venkatesh, V. / Goyal, S. 2011: Expectation Confirmation in Technology Use. Information Systems Research forthcoming.

Craig, G. 2004: Citizenship, Exclusion and Older People. Journal of Social Policy 33(1): 95-114.

Cummings, J. N. / Butler, B. / Kraut, R. 2002: The quality of online social relationships. Communications of the ACM 45(7): 103-108.

Dabholkar, P. A. / Shepherd C. D. / Thorpe, D. I. 2000: A Comprehensive Framework for Service Quality: An Investigation of Critical Conceptual and Measurement Issues Through a Longitudinal Study. Journal of Retailing 76(2): 139-173.

Davis, F. D. / Bagozzi, R. P. / Warshaw, P. R. 1989: User acceptance of computer technology: a comparison of two theoretical models. Management Science 35(8): 982-1003.

Eagle, N. / Pentland, A. 2005: Social Serendipity: Mobilizing Social Software. IEEE Pervasive Computing 4(2): 28-34.

Forum, NFC 2010. NFC Forum: Home. http://www.nfc-forum.org/home/, letzter download am 27.10.2011.

Goyal, S. / Venkatesh, V. 2010: Expectation Disconfirmation and Technology Adoption: Polynomial Modeling and Response Surface Analysis. Management Information Systems Quarterly 34(2): 281-303.

Hawthorn, D. 2003: How universal is good design for older users? 2003 conference on universal usability, Vancouver, Canada: ACM.

Hevner, A. 2007: A Three Cycle View of Design Science Research. Scandinavian Journal of Information Systems 19(2).

House, J. S. 1981: Work Stress and Social Support. Reading, USA: AddisonWesley. 
Joinson, A. N. 2008. Looking at, looking up or keeping up with people?: motives and use of facebook. 26th annual SIGCHI conference on Human factors in computing systems, Florence, Italy: ACM.

Köbler, F. / Koene, P. / Krcmar, H. / Altmann, M / Leimeister, J. M. 2010: LocaTag - An NFC-Based System Enhancing Instant Messaging Tools with Real-Time User Location. Second International Workshop on Near Field Communication (NFC), Monaco, Monte Carlo.

Koene, P. / Köbler, F. / Burgner, P. / Resatsch, R. / Sandner, U. / Leimeister, J. M. / Krcmar, H. 2010: RFID-Based Media Usage Panels in Real-World Settings. 18th European Conference on Information Systems, Pretoria, SA: Department of Informatics.

Kostakos, V. / O’Neill, E. 2008: Cityware: Urban computing to bridge online and real-world social networks. In: Foth, M. (Hg.): Handbook of Research on Urban Informatics: the Practice and Promise of the Real-Time City. Hershey, USA: Information Science Reference, IGI Global, 196-205.

Lampe, C. / Ellison, N. / Steinfield, C. 2006: A face(book) in the crowd: social Searching vs. social browsing. 20th anniversary conference on Computer supported cooperative work, Banff, Canada: ACM.

Lampe, C. / Ellison, N. / Steinfield, C. 2007: A familiar face(book): profile elements as signals in an online social network. SIGCHI conference on Human factors in computing systems, San Jose, USA: ACM.

Leimeister, J. M. / Daum, M. / Krcmar, H. 2004: Towards mobile communities for cancer patients: the case of krebsgemeinschaft.de. International Journal of Web Based Communities 1(1): 58-70.

Leimeister, J. M. / Glauner, C. 2008: Hybride Produkte - Einordnung und Herausforderungen für die Wirtschaftsinformatik. WIRTSCHAFTSINFORMATIK 50(3): 248-251.

Leimeister, J. M. / Schweizer, K. / Leimeister, S. / Krcmar, H. 2008: Do virtual communities matter for the social support of patients?: Antecedents and effects of virtual relationships in online communities. Information Technology \& People 21(4): 350 - 374.

Madlmayr, G. / Langer, J. / Kantner, C. / Scharinger, J. 2008: NFC Devices: Security and Privacy. Availability, Reliability and Security, International Conference on, Los Alamitos, USA: IEEE Computer Society.

Mulliner, C. 2009: Vulnerability Analysis and Attacks on NFC-Enabled Mobile Phones. Availability, Reliability and Security, International Conference on, Los Alamitos, USA: IEEE Computer Society.

Oliver, R. L. 1980: A Cognitive Model of the Antecedents and Consequences of Satisfaction Decisions. Journal of Marketing Research 17(4): 460-469. 
Ondrus, J. / Pigneur, Y. 2007: An Assessment of NFC for Future Mobile Payment Systems. Mobile Business, International Conference on, Los Alamitos, USA: IEEE Computer Society.

Parks, M. R. / Floyd, K. 1996: Making Friends in Cyberspace. Journal of Communication 46(1): 80-97.

Parks, M. R. / Roberts, L.D. 1998: 'Making Moosic': The Development of Personal Relationships on Line and a Comparison to their Off-Line Counterparts. Journal of Social and Personal Relationships 15(4): 517-537.

Patterson, P. G. / Johnson, L. W. / Spreng, R. A. 1997: Modeling the Determinants of Customer Satisfaction for Business-to-Business Professional Services. Journal of the Academy of Marketing Science 25(1): 4-17.

Peffers, K. / Tuunanen, T. / Gengler, C. E. / Rossi, M. /Hui, W. / Virtanen, V. / Bragge, J. 2006: The design science research process: a model for producing and presenting information systems research. First International Conference on Design Science Research in Information Systems and Technology (DERIST), Claremont, USA.

Persson, P. / Blom, J. / Jung, Y. 2005: DigiDress: A Field Trial of an Expressive Social Proximity Application. In: Beigl, M. (Hg.): UbiComp 2005, LNCS 3660. Berlin, Heidelberg: Springer-Verlag, 195-212.

Pietiläinen, A.-K. / Oliver, E. / Lebrun, J. / Varghese, G. / Diot, C. 2009: MobiClique: Middleware for Mobile Social Networking. 2nd ACM workshop on Online social networks, Barcelona, Spain: ACM.

Pizam, A. / Milman, A. 1993: Predicting satisfaction among first time visitors to a destination by using the expectancy disconfirmation theory. International Journal of Hospitality Management 12(2): 197-209.

Plos, O. / Buisine, S. 2006: Universal design for mobile phones. CHI '06 extended abstracts on Human factors in computing systems, Montreal, Canada.

Resatsch, F. 2010: Ubiquitous Computing: Developing and Evaluating Near Field Communication Applications: Gabler, Betriebswirt.-Vlg.

Spreng, R. A. / MacKenzie, S. B. / Olshavsky, R. W. 1996: A Reexamination of the Determinants of Consumer Satisfaction. The Journal of Marketing 60(3): 15-32.

Staples, D. S. / Wong, I. / Seddon, P. B. 2002: Having expectations of information systems benefits that match received benefits: does it really matter? Information and Management 40: 115-131.

Stuck, A. E. /Walthert, J. M. / Nikolaus, T. / Büla, C. J. / Hohmann, C. / Beck, J. C. 1999: Risk factors for functional status decline in community-living elderly 
people: a systematic literature review. Social Science \& Medicine 48(4): 445469.

Swan, J. E. / Trawick, I. F. 1981: Disconfirmation of expectations and satisfaction with a retail service. Journal of Retailing 57(Fall): 49-67.

Szajna, B. / Scamell, R. W. 1993: The Effects of Information System User Expectations on Their Performance and Perceptions. MIS Quarterly 17(4): 493516.

Tan, B. C. Y. / Wei, K.-w., Sia, C.-L. / Raman, K. S. 1999: A partial test of the task-medium fit proposition in a group support system environment. $A C M$ Transactions on Computer-Human Interaction (TOCHI) 6: 47-66.

Tse, D. K. / Wilton, P. C. 1988: Models of Consumer Satisfaction Formation: An Extension. Journal of Marketing Research 25(2): 204-212.

Vasalou, A. / Joinson, A. N. / Courvoisier, D. 2010: Cultural differences, experience with social networks and the nature of "true commitment" in Facebook. International Journal of Human-Computer Studies 68(10): 719-728.

Venkatesh, V. / Davis, F. D. 2000: A Theoretical Extension of the Technology Acceptance Model: Four Longitudinal Field Studies. Management Science 46(2): 186-204.

Venkatesh, V. / Morris, M. G. / Gordon, B. D. / Davis, F. D. 2003: User Acceptance of Information Technology: Toward a Unified View. MIS Quarterly 27(3): 425-478.

Wellman, B. / Quan Haase, A. / Witte, J. / Hampton, K. 2001: Does the Internet Increase, Decrease, or Supplement Social Capital?: Social Networks, Participation, and Community Commitment. American Behavioral Scientist 45(3): 436-455.

Ziv, N. D. / Mulloth, B. 2006: An Exploration on Mobile Social Networking: Dodgeball as a Case in Point. International Conference on Mobile Business, Copenhagen, Denmark: IEEE Computer Society. 